\title{
Optimal Planning of Charging Station for Phased Electric Vehicle*
}

\author{
Yajing Gao, Yandong Guo \\ School of Electrical and Electronic Engineering, North China Electric Power University, Baoding, China \\ Email: commoncat@163.com \\ Received March, 2013
}

\begin{abstract}
Construction of electric vehicle charging station is the premise of the popularity of electric vehicles. In this paper, electric vehicle charging facilities planning is divided into two stages: public demonstration stage and commercial operation stage. In public demonstration stage, This Paper applies maximal covering model into charging station location, and then be solved by the branch and bound method. In commercial operation stage, the charging station's service areas division is studied based on Voronoi diagram. With the concerning technical restrains, an optimal planning method is presented for newly-increased station positioning and service region division. The method using the most greatly air circuit method and local dynamic characteristics of Voronoi diagram guarantees the reasonable distribution of stations. Then charging station model of optimal load allocation is established by using $M / M / c$ model. In the end, the correctness and validity of the model is verified through simulation of charging station planning.
\end{abstract}

Keywords: Charging Demand; Electric Vehicle; Planning Layout; Voronoi

\section{Introduction}

With the deteriorating global environment and the lack of oil resources, the society has begun to focus on the use of electric vehicles. However, construction of electric vehicle charging station is the premise of the popularity of electric vehicles [1-3].

At present, construction of charging stations in China is mainly for market demonstration with no clear and reasonable layout, so establishing an effective charging station layout and a scale setting tool have become an urgent need at the moment. In [4], the advantages and disadvantages of the electric vehicle charging system have been reviewed and the external access methods of charging stations, installed capacity and its influencing factors are considered as a preliminary inquiry for the planning of charging stations. In [5], the overall demand of the charging load and planning influencing factors, including the operating model of electric vehicles charging stations have been analyzed; principle recommendations such as planning of charging stations should meet the radius requirements of the charging stations, traffic density, distribution of electric vehicle charging demand and the overall urban road planning have been made. In [6], taking the geographical factors and the service radius

\footnotetext{
*This work was supported in part by the National Natural Science Foundation of China under Grant 51177047, Fundamental Research Funds for the Central Universities under Grant 12MS107.
}

into account, a two-step screening method to identify candidate sites for charging stations has been presented.

Combined with various sections of the electric vehicle driving conditions and charging needs, in public demonstration stage maximum coverage model has been presented to strike the optimal location under funding constraints; in commercial operation stage, Voronoi diagram is used to distribute demand, an optimized model is presented.

\section{Analysis of Demand for Electric Vehicle Charging}

Assuming vehicle charging demand is equal the power consumption on the section $L$.

$V(i, k)$ Calculate the class-k electric vehicle electric consumption in $V(i, k)$ on the section $L(i)$ :

$$
V(i, k)=g(k) \times L(i) \times q(i, k)
$$

The power consumption of various types of electric vehicles on the section of $L(i)$ :

$$
V(i)=\sum_{k=1}^{m} V(i, k)
$$

In the equation (1) (2): $g$ is electricity consumption of vehicles per kilometer constrained by vehicle type, vehicle load and travel speed. This model uses the concept of the average electricity consumption for a particular class 
of electric vehicles and average power consumption is a fixed value; $q(i)$ is the number of vehicles through a road section in a unit time (vehicles / day). $Q(i, k)$ is the traffic volume at point I for class- $k$ cars in a two-way traffic flow; $L(i)$ is the length of the road section $i$.

\section{Charging Station Planning in Public Demonstration Stage}

The current stage of electric vehicle technology is not yet fully mature and effective and sustainable market mechanism to promote the development of electric vehicles has not yet formed. The total amount of electric vehicles accounts for a very low proportion and electric vehicle industry relies on government subsidies and advocacy, in the public demonstration stage, charging facilities planning can be seen as a short-term planning.

Because of the characteristics of this stage, the initial investment of charging stations covering all charging needs may lead to excessive fiscal spending. As a result, due to the limitations of the actual budget may, charging stations may cover less demand. The model is shown as follows:

$$
\begin{gathered}
\max Z=\sum_{i \in I} V(i) m_{i} \\
\text { s.t. } \quad m_{i} \leq \sum_{i \in N_{i}} n_{j}, i \in I \\
\sum_{j \in J} n_{j}=p \\
n_{j}, m_{i} \in\{0,1\} \quad i \in I, j \in J
\end{gathered}
$$

In the model, $J$ is a set of candidate points of existing gas station scalable with charging facilities and $I$ is a set of charging requirements. $m_{\mathrm{i}}$ stands for whether charging demand point $i$ could receive charging services and the value of 1 shows charging demand in point $i$ can receive charging service, on contrary the value is zero. $N_{i}$ is a set of candidate facility $j$ which could cover charging demand in point $i$ and it can be expressed as $N_{i}=$ $\left\{j \mid d_{i j} \leq s\right\}$, where $d$ stands for distance between point $i$ and $j$ and $s$ stands for the maximum acceptable candidate facility charge distance. The objective function of formula (3) targets to covering most of the charging requirements by charging facilities with the $p$; constraint condition Equation (4) indicates that the number that charging stations can be searched by charging demand point $i$ within a maximum acceptable service distance is greater or equal with the value of 1 ; constraint formula (5) represents the total number of facilities is limited to the number of $p$. The constraint formula (6) constrains the value of $m_{i}$ and $n_{j}$ to the value of 0 or 1 .

Described by the formula (3) (6), the charging station planning problem is a $0-1$ integer programming problem and will be solved by the branch and bound method in the follow.

\section{Charging Station Planning in Commercial Operation Stage}

In this stage, the electric vehicle technology is assumed basically mature and the total amount of vehicles reaches a certain size. So in this stage, electric vehicle charging facilities planning can be seen as a long-term planning.

\subsection{Method of Alternately Positioning in Commercial Operation Stage}

In this stage, adequate charging facilities begin to be established to meet the charging demand of electric vehicle. Assume that electric car owners are always looking for the nearest charging station for their services, the assumption coordinates a great similarity with the Voronoi diagram [7]. Program flow of alternately positioning in commercial operation stage is shown in Figure 1:

Method of alternately positioning in commercial operation stage is as follows:

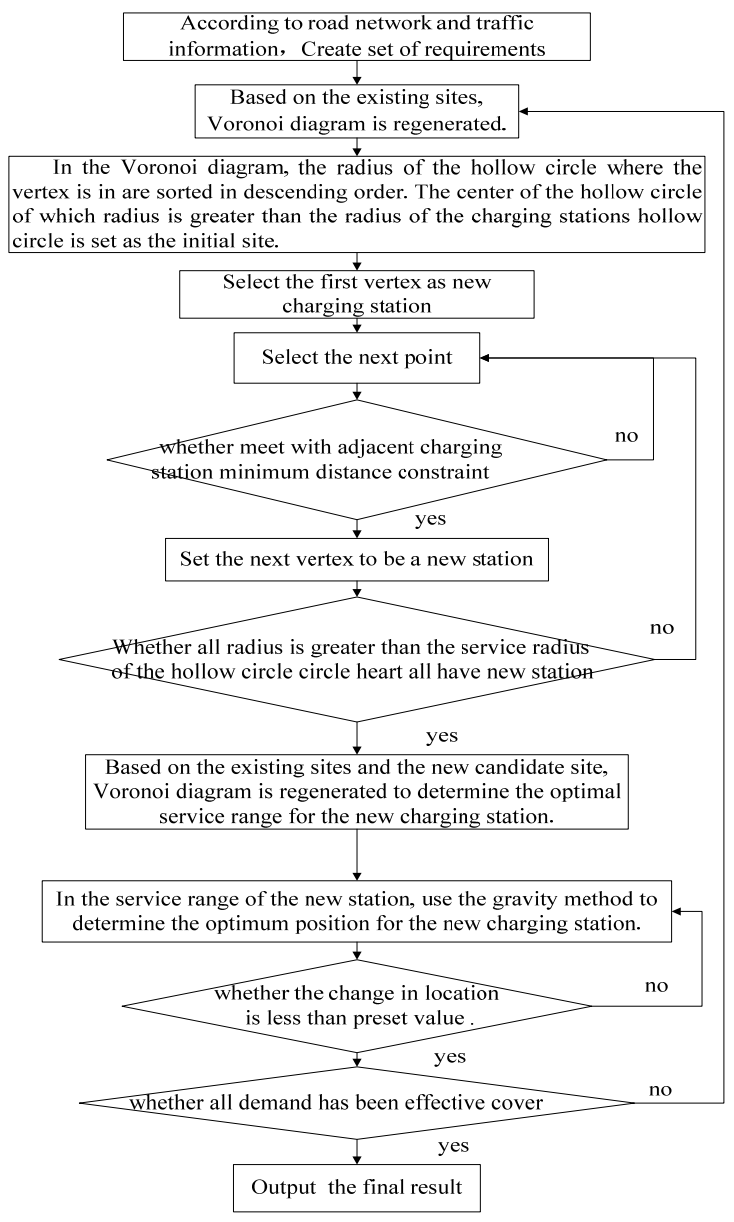

Figure 1. Flow charts. 
1) Using charging station sites in the first stage, the Voronoi diagram is generated. In the Voronoi diagram, the radius of the hollow circle where the vertex in are sorted in descending order. The center of the hollow circle of which radius is greater than the radius of the charging stations hollow circle is set as the initial site.

2) Based on the existing sites and the new candidate site, Voronoi diagram is regenerated to determine the optimal service range for the new charging station. In the service range of the new station, Use the gravity method to determine the optimum position for the new charging station.

3) Repeat 2) until the value of location coordinate of the new sites is less than booking precision.

\subsection{The Number of the Optimal Chargers in Charging Station}

From the current developing situation of electric vehicle and historical data at gas stations, the following assumptions are made:

Per charge quantity is a fixed value $Q$. The arrivals of electric vehicles are a Poisson stream of rate $\lambda$. The charging time is a negative exponential distribution with $\mu$.

With the above assumptions, the charging station, as a charging service system, is a multi service desk and finite waiting room $(M / M / c)$ system.

There are $i$ sections in area $j, V_{j}$ is the electric consumption in area $j$ :

$$
V_{\mathrm{j}}=\sum_{\mathrm{i}=1}^{\mathrm{i}} V(i)
$$

$N$ is the number of times of electric vehicles charge in area $i$

$$
N=V_{\mathrm{j}} / Q
$$

Assuming vehicle charging station is open from 8 am to $10 \mathrm{pm}$.

$$
\lambda=N / 14
$$

The model of optimizing the quantity of chargers in charging stations is as follow:

$$
\begin{gathered}
\min F_{i}=c \times T \frac{r_{0}\left(1+r_{0}\right)^{n}}{\left(1+r_{0}\right)^{n}-1}+Y_{i}+N \times k \times W_{q} \\
\mathrm{~L}_{\mathrm{q}} \leq L_{\max }
\end{gathered}
$$

In (10), $F_{i}$ is the cost of the charging station $I, c$ is the number of chargers, $T$ is the charger cost, $Y_{i}$ is the annual operating cost of the charging station $i . k$ is the user's time value, it can be estimated by the user's income in the area. $n$ is the depreciable life, $W_{q}$ is the average wait time. In (11), $L_{q}$ is the mathematical expectation of the number of waiting EVs, $L_{\max }$ is the maximum acceptable number of waiting EVs in station.

The problem will be solved by the LINGO in the follow.

\section{Example Analysis}

In this paper, planning of charging stations is taken for example: the region is a total area of $36.48 \mathrm{~km}^{2}$ which spans $6.4 \mathrm{~km}$ from east to west and $5.7 \mathrm{~km}$ from north to south and can be divided into 58 sections. In reality, the type of electric vehicles differ from driving load and traffic status, charge cost will also be different. However, to simplify the problem, the example assumes that power consumption of vehicles per kilometer is a fixed value, $g$ $=0.13 \mathrm{kWh} / \mathrm{km}$. The map as well as the existing gas station location with link numbers is shown in Figure 2.

\subsection{Charging Station Planning in Public Demonstration Stage}

The region is planned to construct 5 charging stations in the first batch and the predetermined range of the charging station is $2 \mathrm{~km}$. According to the data in the Equation (1)(2), the charging demands of different sections are obtained. According to the charging station planning model in public demonstration stage constrained by Equation (3) (6), the demand is set to weight, then solve by branch and bound method in lindo software. The max $Z$ is $14862 \mathrm{kWh}$ and the most optimal position is shown in Figure 3. By the optimal location, Voronoi diagram is generated, the scope of each charging station is determined and each demand point is allocated, shown in Figure 3.

\subsection{Charging Station Planning in Commercial Operation Stage}

In Figure 3, the hollow circle radius greater than the radius of the charging stations hollow circle center are set as the initial location. Using the existing stations with

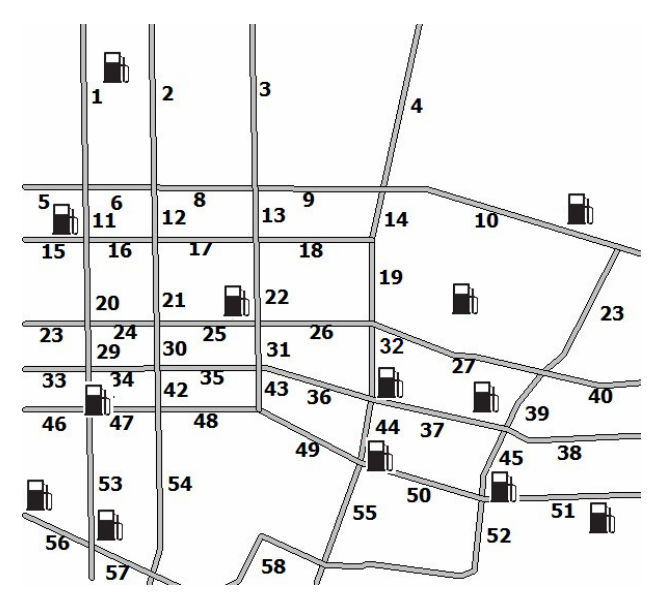

Figure 2. City network diagram. 
new sites to generate Voronoi diagram, the scope of services of the new station is determine, in other words, demand is distributed again. Within the range of its services, Use the gravity method to determine the optimum position for the new charging station. Using the existing stations with new optimal site to generate new Voronoi diagram and solve the problem again with the new service scope division of charging stations until the iterative adjustment is less than the default precision of $0.1 \mathrm{~km}^{2}$. The location of the new station and its service range is shown in Figure 4. The new site is located in point 6 .

\subsection{The Number of the Optimal Chargers in Charging Station}

Taking into account the interests of charging stations and users, the sum of charging stations' service cost and customers' waiting fees is chosen as the objective function for the optimal allocation of chargers.

Relevant parameters are shown in Table 1.

Using the methods described in this article, the problem has been calculated by LINGO, and then following results have been obtained in Table 2 .

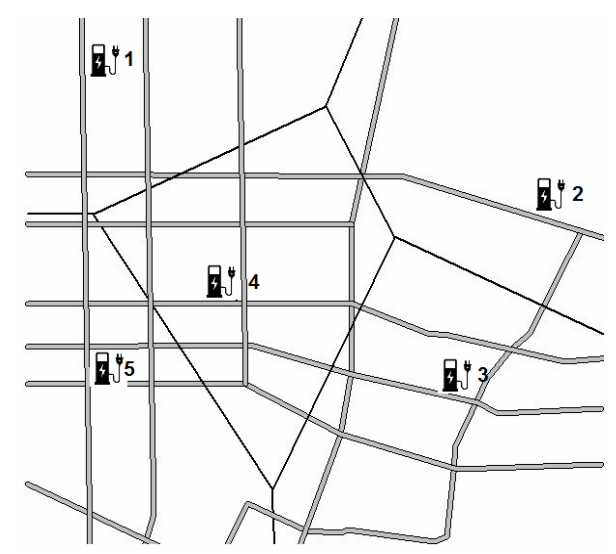

Figure 3. Charging station planning in public demonstration stage.

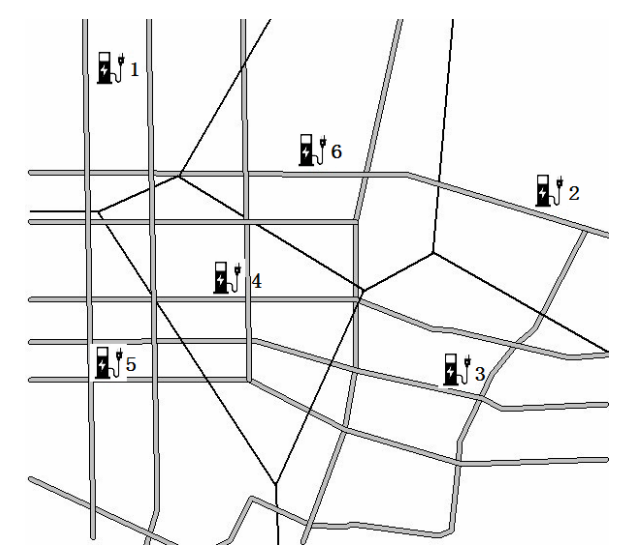

Figure 4. Charging station planning in commercial operation stage.
Table 1. Related parameter selection.

\begin{tabular}{cccc}
\hline sign & Value & sign & Value \\
\hline$Q$ & $20.8 \mathrm{kWh}$ & $g$ & $0.13 \mathrm{kWh} / \mathrm{km}$ \\
$L_{\max }$ & 20 & $r^{0}$ & 0.1 \\
$T$ & 0.1 million yuan & $k$ & 25 yuan $/$ hour \\
$\mathrm{Y}_{\mathrm{i}}$ & 0.2 million yuan & $n$ & 20 \\
\hline
\end{tabular}

Table 2. The number of optimal chargers.

\begin{tabular}{cc}
\hline Station number & The number of the optimal chargers \\
\hline 1 & 7 \\
2 & 6 \\
3 & 12 \\
4 & 11 \\
5 & 11 \\
6 & 7 \\
\hline
\end{tabular}

\section{Conclusions}

In this paper, using sections consumption to simulate randomly charging requirements of electric vehicles is able to provide new ideas for future research on forecasting electric vehicle charging load; in public demonstration stage, this Paper applies maximal covering model into charging station location, and then be solved by the branch and bound method. In commercial operation stage, using Voronoi diagram features is able to provide automatic positioning of the charging stations and new charging Station by service area division and would also be an attempt to solve the problem of optimized charging station optimized planning; then charging station model of optimal load allocation is established by using $M / M / c$ model. The presented models are verified to be effectiveness through a practical example.

\section{REFERENCES}

[1] Hattonce, Beellask, Brezetjc, et al., "Charging Station for Urban Settings: The Design of a Product Platform for Electric Vehicle Infrastructure in Dutch Citie," World Electric Vehicle Journal, Vol. 3, 2009, pp.1-13.

[2] M. lu, "Home and Abroad Electric Vehicle Charging Facilities Development," Journal of Central China Power, Vol. 5, 2010, pp.16-30.

[3] J. Y. Yao, "Electric Vehicle Charging System Construction Application Analysis," East China electric power, Vol. 4, 2008, pp.107-110.

[4] J. G. Kang, Z. L.Wei, D. M. Cheng, etc., "Electric Vehicle Charging Mode and Charging station Construction Research," Power demand side management, Vol. 5, 2009, pp. 64-66.

[5] F. Xu, G. Q. Yu, L. Gu, etc., "Electric Vehicle Charging 
Station Layout Planning," Journal of East China Electric Power, Vol. 10, 2009, pp.1678-1682.

[6] L. F. Kou, "Regional Electric Vehicle Charging Station Planning Model and Algorithm," Journal of Modern
Electric Power, Vol. 4, 2010, pp. 44-48.

[7] P. D. Zhou, "Computational Geometry-Algorithm Analysis and Design," Beijing: Tsinghua University Press, 2000 . 